\title{
BMJ
}

\section{Cardiovascular safety of non-steroidal anti-inflammatory drugs: network meta-analysis}

\begin{abstract}
Sven Trelle, senior research fellow, ${ }^{1,2}$ Stephan Reichenbach, senior research fellow, ${ }^{1,4}$ Simon Wandel, research fellow, ${ }^{1}$ Pius Hildebrand, clinical reviewer, ${ }^{3}$ Beatrice Tschannen, research fellow, ${ }^{1}$ Peter M Villiger, head of department and professor of rheumatology, ${ }^{4}$ Matthias Egger, head of department and professor of epidemiology and public health, ${ }^{1}$ Peter Jüni, head of division and professor of clinical epidemiology ${ }^{1,2}$
\end{abstract}

\section{ABSTRACT}

Objective To analyse the available evidence on cardiovascular safety of non-steroidal anti-inflammatory drugs.

Design Network meta-analysis.

Data sources Bibliographic databases, conference proceedings, study registers, the Food and Drug Administration website, reference lists of relevant articles, and reports citing relevant articles through the Science Citation Index (last update July 2009). Manufacturers of celecoxib and lumiracoxib provided additional data.

Study selection All large scale randomised controlled trials comparing any non-steroidal anti-inflammatory drug with other non-steroidal anti-inflammatory drugs or placebo. Two investigators independently assessed eligibility.

Data extraction The primary outcome was myocardial infarction. Secondary outcomes included stroke, death from cardiovascular disease, and death from any cause. Two investigators independently extracted data.

Data synthesis 31 trials in 116429 patients with more than 115000 patient years of follow-up were included. Patients were allocated to naproxen, ibuprofen, diclofenac, celecoxib, etoricoxib, rofecoxib, lumiracoxib, or placebo. Compared with placebo, rofecoxib was associated with the highest risk of myocardial infarction (rate ratio $2.12,95 \%$ credibility interval 1.26 to 3.56 ), followed by lumiracoxib $(2.00,0.71$ to 6.21$)$. Ibuprofen was associated with the highest risk of stroke $(3.36,1.00$ to 11.6$)$, followed by diclofenac $(2.86,1.09$ to 8.36$)$. Etoricoxib $(4.07,1.23$ to $15.7)$ and diclofenac $(3.98,1.48$ to 12.7$)$ were associated with the highest risk of cardiovascular death.

Conclusions Although uncertainty remains, little evidence exists to suggest that any of the investigated drugs are safe in cardiovascular terms. Naproxen seemed least harmful. Cardiovascular risk needs to be taken into account when prescribing any non-steroidal antiinflammatory drug.

\section{INTRODUCTION}

Non-steroidal anti-inflammatory drugs (NSAIDs) have been the cornerstone of pain management in patients with osteoarthritis and other painful conditions. In the United States an estimated 5\% of all visits to a doctor are related to prescriptions of non-steroidal anti-inflammatory drugs and they are among the most commonly used drugs. ${ }^{12}$ In 2004, rofecoxib, marketed as a cyclooxygenase-2 (COX 2) selective inhibitor, was withdrawn from the market after the results of a randomised placebo controlled trial ${ }^{3}$ showed an increased risk of cardiovascular events associated with the drug. This finding was confirmed in other trials and a cumulative meta-analysis. ${ }^{4}$ Since then debate has surrounded the cardiovascular safety of cyclo-oxygenase- 2 selective inhibitors, followed by similar concerns about traditional non-steroidal anti-inflammatory drugs. ${ }^{5}$ More recently, the US Food and Drug Administration decided against the approval of etoricoxib because of its inadequate risk-benefit profile. ${ }^{6}$

These debates and the patchwork of evidence resulting from multiple trials and cohort studies have unsettled practising clinicians. ${ }^{7}$ Several standard meta-analyses were unable to resolve the debate because they failed to integrate all available randomised evidence in one analysis. Network meta-analysis allows a unified, coherent analysis of all randomised controlled trials that compare non-steroidal antiinflammatory drugs head to head or with placebo while fully respecting randomisation. ${ }^{89} \mathrm{We}$ analysed the cardiovascular safety of non-steroidal anti-inflammatory drugs by integrating all available direct and indirect evidence in network meta-analyses.

\section{METHODS}

We considered large scale randomised controlled trials comparing any non-steroidal anti-inflammatory drug with other non-steroidal anti-inflammatory drugs, paracetamol (acetaminophen), or placebo for any medical condition. To be included, trials required at least two arms with at least 100 patient years of follow-up. In the case of trials with several arms, we included only arms with at least 100 patient years of follow-up. We excluded trials in patients with cancer. For an intervention to be included in our analyses, at least 10 patients allocated to 
the intervention had to have had a myocardial infarction in all eligible trials combined.

\section{Trial identification and data collection}

We searched bibliographic databases, relevant conference proceedings, study registers, and the FDA website, manually searched reference lists of relevant articles, and retrieved reports citing relevant articles through the Science Citation Index (see web extra appendix 1). The search was last updated in July 2009. Two investigators independently assessed trials for eligibility and extracted data. If a trial was covered in more than one report we used a hierarchy of data sources: reports to the FDA, peer reviewed articles, reports from the web based repository for results of clinical studies www.clinicalstudyresults.org, published abstracts, and other sources, such as trial websites. Finally, we contacted all authors of primary trial reports and manufacturers of relevant non-steroidal anti-inflammatory drugs (Pfizer, Merck, Novartis) for missing outcome data. One independent investigator ${ }^{10}$ and two manufacturers (Pfizer and Novartis) provided additional information.

\section{Outcome measures}

The prespecified primary outcome was fatal or nonfatal myocardial infarction. Secondary outcomes were haemorrhagic or ischaemic fatal or non-fatal stroke; cardiovascular death, defined as any death due to cardiovascular causes (for example, myocardial infarction, low output failure, fatal arrhythmia, pulmonary embolism, stroke), and death of unknown cause; death from any cause; and the Antiplatelet Trialists' Collaboration composite outcome ${ }^{11}$ of non-fatal myocardial infarction, non-fatal stroke, or cardiovascular death.

\section{Statistical analysis}

Whenever possible we used results from intention to treat analysis of the longest follow-up available. We excluded comparisons with zero events in both groups from the relevant analysis since such comparisons provide no information on the magnitude of the treatment effect. ${ }^{12}$ We used a Bayesian random effects model, which fully preserves randomised treatment comparisons within trials. ${ }^{91314}$ Analyses were done using Markov chain Monte Carlo methods with minimally informative prior distributions. As measures of treatment effects, we calculated rate ratios based on patient years. We estimated rate ratios from the median of the posterior distribution as well as corresponding 95\% credibility intervals. In the presence of minimally informative priors, credibility intervals can be interpreted like conventional confidence intervals. Rate ratios below 1 indicate a detrimental effect of the control intervention throughout. Finally, we calculated confidence levels, defined as the posterior probability that an increase in risk is smaller than a specified threshold. ${ }^{15}$ Confidence levels take into account both the magnitude of the pooled rate ratio and the corresponding uncertainty. Precise estimates are more informative and result in sharp increases in the confidence that the rate ratio of a drug does not exceed a specified threshold. When the specified threshold of the rate ratio increases, imprecise estimates that are based on low numbers of events are uninformative and lead to slow increases in confidence and relevant uncertainty even for large rate ratios. We prespecified a rate ratio of 1.3 as the primary threshold, which was used as non-inferiority margin in the Multinational Etoricoxib and Diclofenac Arthritis Long-term (MEDAL) programme. ${ }^{16}$ We used linear regression to test for each outcome whether there was any association with cyclo-oxygenase- 2 selectivity (data only shown in web extra appendix 2).

We assessed the goodness of fit of the model to the data by calculating the residual deviance ${ }^{9}$ the heterogeneity of treatment effects estimated from the median between trial variance $\tau^{2}$ observed in the posterior distribution, and the consistency of the network determined by use of inconsistency factors, ${ }^{17}$ defined as the difference in $\log$ rate ratios derived from direct and indirect comparisons. To check the robustness of our analyses, we calculated Bayesian random effects meta-analyses for all available direct comparisons and carried out several sensitivity analyses, including a restriction to trials in patients with musculoskeletal conditions (data shown in web extra appendix 2). For all analyses we used Stata release 10, and WinBUGS version 1.4 (MRC Biostatistics Unit, Cambridge, UK). Web extra appendix 1 provides further details of the methods used.

\section{RESULTS}

Thirty one randomised controlled trials evaluating seven different non-steroidal anti-inflammatory drugs were included in the analyses (table 1, fig 1). Celecoxib

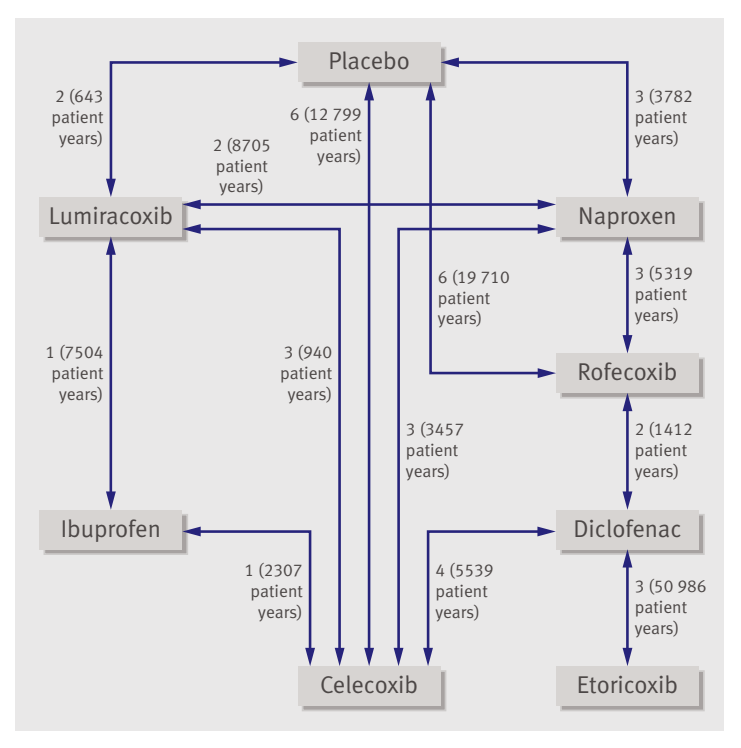

Fig 1| Network of comparisons included in analyses. Solid lines represent direct comparisons within randomised controlled trials. Numbers denote trials comparing corresponding interventions, with overall number of patient years of follow-up in brackets 
was investigated most (15 trials) and compared with five different interventions. Ibuprofen was evaluated least (two trials) and compared with two different interventions, whereas etoricoxib was evaluated in three trials but compared with only one intervention. Etoricoxib and diclofenac had the largest number of patient years of follow-up (26 025 and 27819 overall, respectively), whereas ibuprofen had the lowest number of patient years of follow-up (4832 overall). In total, 116429 patients with 117218 patient years of followup were covered in the analysis of the primary outcome (table 2). The methodological quality of included trials was generally high with all but two having adequate concealment of allocation, all having adequate blinding of patients and investigators, 16 having independent event adjudication, and 13 including all randomised patients in the analysis (table 1).

\section{Myocardial infarction}

Twenty nine trials with 554 accumulated events contributed to the analysis of myocardial infarction (table 2). For three of the preparations (naproxen, diclofenac, and etoricoxib) evidence was lacking for an increased risk of myocardial infarction compared with placebo (fig 2). All other drugs seemed to be associated with an increased risk compared with placebo. Estimated rate ratios were greater than 1.3 for ibuprofen $(1.61,95 \%$ credibility interval 0.50 to 5.77$)$, celecoxib $(1.35,0.71$ to 2.72$)$, rofecoxib $(2.12,1.26$ to $3.56)$, and lumiracoxib (2.00, 0.71 to 6.21$)$.

\section{Stroke}

Twenty six trials with 377 accumulated events contributed to the analysis of stroke (table 2). All drugs seemed to be associated with an increased risk compared with placebo (fig 2). Estimated rate ratios were greater than 1.3 for naproxen $(1.76,0.91$ to 3.33$)$, ibuprofen $(3.36,1.00$ to 11.60$)$, diclofenac $(2.86,1.09$ to $8.36)$, etoricoxib (2.67, 0.82 to 8.72 ), and lumiracoxib $(2.81,1.05$ to 7.48$)$.

\section{Cardiovascular death}

Twenty six trials with 312 accumulated events contributed to the analysis of cardiovascular death, accounting for $46 \%$ of all deaths (table 2). All drugs except naproxen showed some evidence for an increased risk of cardiovascular death compared with placebo (fig 2). The estimated rate ratios for cardiovascular death were greater than 1.3 for ibuprofen $(2.39,0.69$ to 8.64$)$, diclofenac $(3.98,1.48$ to 12.70$)$, celecoxib (2.07, 0.98 to 4.55$)$, etoricoxib $(4.07,1.23$ to 15.70$)$, rofecoxib (1.58, 0.88 to 2.84$)$, and lumiracoxib (1.89, 0.64 to 7.09$)$.

\section{Death from any cause}

Twenty eight trials with 676 accumulated events contributed to the analysis on overall mortality (table 2). All the drugs seemed to be associated with increased risks of death from any cause compared with placebo (fig 2). The estimated rate ratios were greater than 1.3

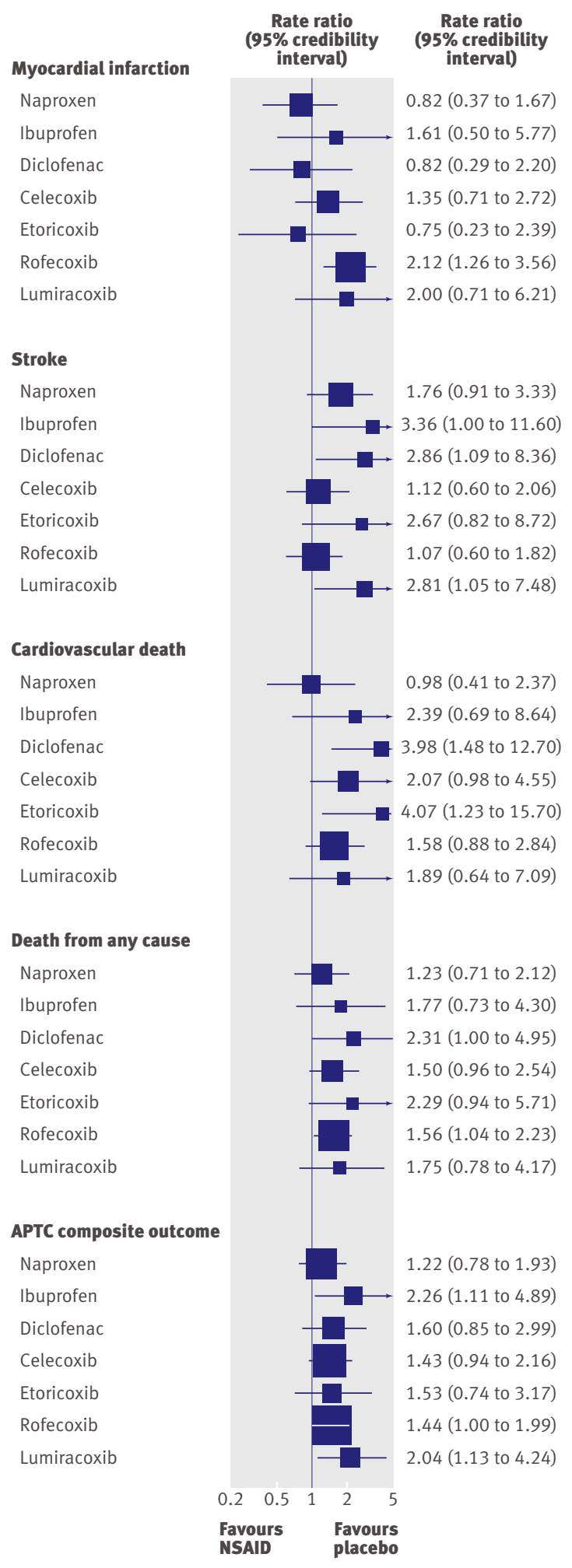

Fig 2 | Estimates of rate ratios for non-steroidal antiinflammatory drugs compared with placebo. NSAID=nonsteroidal anti-inflammatory drug; APTC=Antiplatelet Trialists' Collaboration

for ibuprofen (1.77, 0.73 to 4.30), diclofenac (2.31, 1.00 to 4.95$)$, celecoxib (1.50, 0.96 to 2.54$)$, etoricoxib (2.29, 0.94 to 5.71$)$, rofecoxib $(1.56,1.04$ to 2.23$)$, and lumiracoxib $(1.75,0.78$ to 4.17$)$. 
Table 1/Characteristics of included trials

\begin{tabular}{|c|c|c|c|c|c|c|c|c|c|c|}
\hline Trial & Interventions & Population & $\begin{array}{c}\text { No of } \\
\text { patients* }\end{array}$ & $\begin{array}{l}\text { Follow-up } \\
\text { (weeks) }\end{array}$ & $\begin{array}{l}\text { Low dose } \\
\text { aspirin } \\
\text { allowed }\end{array}$ & $\begin{array}{l}\text { Concealed } \\
\text { allocation† }\end{array}$ & Blinding $\ddagger$ & $\begin{array}{l}\text { Events } \\
\text { adjudica- } \\
\text { ted§ }\end{array}$ & $\begin{array}{l}\text { Intention to } \\
\text { treat }\end{array}$ & Source \\
\hline ADAPT & $\begin{array}{l}\text { Placebo } v \text { naproxen }(440 \mathrm{mg} / \mathrm{d}) v \\
\text { celecoxib }(400 \mathrm{mg} / \mathrm{d})\end{array}$ & $\begin{array}{l}\text { At risk for Alzheimer } \\
\text { disease }\end{array}$ & 2528 & 198 & Yes & Yes & Yes & Yes & Yes & Journal article, ${ }^{\mathrm{w} 1}$ trial website ${ }^{\mathrm{w} 2}$ \\
\hline Aisen 2003 & $\begin{array}{l}\text { Placebo } v \text { naproxen }(440 \mathrm{mg} / \mathrm{d}) v \\
\text { rofecoxib }(25 \mathrm{mg} / \mathrm{d})\end{array}$ & Alzheimer disease & 351 & 60 & Yes & Yes & Yes & No & Yes & $\begin{array}{l}\text { Journal article, }{ }^{\mathrm{w} 3} \text { personal } \\
\text { communication }^{\mathrm{w} 4}\end{array}$ \\
\hline Geusens 2004 & $\begin{array}{l}\text { Placebo } v \text { naproxen }(1000 \mathrm{mg} / \mathrm{d}) \\
\text { v lumiracoxib }(200,400 \mathrm{mg} / \mathrm{d})\end{array}$ & $\begin{array}{l}\text { Rheumatoid } \\
\text { arthritis }\end{array}$ & 1124 & 26 & Yes & Yes & Yes & No & Yes & $\begin{array}{l}\text { Journal article, }{ }^{\mathrm{w} 5} \text { personal } \\
\text { communication }^{\mathrm{w} 6 \mathrm{w} 7}\end{array}$ \\
\hline APC & $\begin{array}{l}\text { Placebo } v \text { celecoxib ( } 400 \text {, } \\
800 \mathrm{mg} / \mathrm{d})\end{array}$ & $\begin{array}{l}\text { Adenomatous } \\
\text { polyps (colon) }\end{array}$ & 2035 & 160 & Yes & Yes & Yes & Yes & Yes & Journal article, ${ }^{\text {w8 }}$ study register ${ }^{\mathrm{w} 9}$ \\
\hline GAIT & Placebo $v$ celecoxib $(200 \mathrm{mg} / \mathrm{d})$ & Osteoarthritis & 631 & 24 & No & Yes & Yes & No & Yes & Journal article $\mathrm{w}^{\mathrm{w} 10}$ \\
\hline IQ5-97-02-001 & Placebo $v$ celecoxib $(400 \mathrm{mg} / \mathrm{d})$ & $\begin{array}{l}\text { At risk for Alzheimer } \\
\text { disease }\end{array}$ & 425 & 52 & Yes & Yes & Yes & No & No & $\begin{array}{l}\text { FDA reports, }{ }^{\mathrm{w} 11} \text { study register, }{ }^{\mathrm{w} 12} \\
\text { personal communication }{ }^{\mathrm{w} 13} \text { w14 }\end{array}$ \\
\hline PreSAP & Placebo $v$ celecoxib $(400 \mathrm{mg} / \mathrm{d})$ & $\begin{array}{l}\text { Adenomatous } \\
\text { polyps (colon) }\end{array}$ & 1561 & 159 & Yes & Yes & Yes & Yes & Yes & $\begin{array}{l}\text { Journal article, }{ }^{\text {w15 }} \text { personal } \\
\text { communication }^{\text {w13 w14 }}\end{array}$ \\
\hline Lehmann 2005 & $\begin{array}{l}\text { Placebo } v \text { celecoxib }(200 \mathrm{mg} / \mathrm{d}) v \\
\text { lumiracoxib }(100 \mathrm{mg} / \mathrm{d})\end{array}$ & Osteoarthritis & 1684 & 13 & Yes & Yes & Yes & Yes & Yes & $\begin{array}{l}\text { Journal article, }{ }^{\mathrm{w} 16} \text { personal } \\
\text { communication }^{\mathrm{w} 6 \mathrm{w7}}\end{array}$ \\
\hline APPROVe & Placebo $v$ rofecoxib $(25 \mathrm{mg} / \mathrm{d})$ & $\begin{array}{l}\text { Adenomatous } \\
\text { polyps (colon) }\end{array}$ & 2586 & 229 & Yes & Unclear & Yes & Yes & Yes & Journal article ${ }^{\mathrm{w} 17}$ \\
\hline Reines 2004 & Placebo $v$ rofecoxib $(25 \mathrm{mg} / \mathrm{d})$ & Alzheimer disease & 692 & 65 & Unclear & Yes & Yes & Yes & Yes & FDA reportsw18 $^{\text {w18 }}$ \\
\hline Thal 2005 & Placebo $v$ rofecoxib $(25 \mathrm{mg} / \mathrm{d})$ & $\begin{array}{l}\text { At risk for Alzheimer } \\
\text { disease }\end{array}$ & 1457 & 210 & Yes & Yes & Yes & Yes & Yes & Journal article ${ }^{\mathrm{w} 19}$ \\
\hline VICTOR & Placebo $v$ rofecoxib $(25 \mathrm{mg} / \mathrm{d})$ & $\begin{array}{l}\text { Adjuvant (colon } \\
\text { cancer) }\end{array}$ & 2327 & 235 & Yes & Yes & Yes & Yes & No & $\begin{array}{l}\text { FDA reports, }{ }^{1618} \text { journal article, }{ }^{\text {w20 }} \\
\text { study register, }{ }^{\text {w21 }} \text { trial website }^{\text {w22 }}\end{array}$ \\
\hline ViP & Placebo $v$ rofecoxib $(25 \mathrm{mg} / \mathrm{d})$ & $\begin{array}{l}\text { At risk for prostate } \\
\text { cancer }\end{array}$ & 4741 & 75 & Yes & Yes & Yes & Yes & Yes & Study register ${ }^{\mathrm{w} 23}$ \\
\hline A3191152 & $\begin{array}{l}\text { Naproxen }(1000 \mathrm{mg} / \mathrm{d}) v \\
\text { celecoxib }(200 \mathrm{mg} / \mathrm{d})\end{array}$ & Osteoarthritis & 589 & 26 & Yes & Yes & Yes & No & No & Study register ${ }^{\mathrm{w} 24}$ \\
\hline $\begin{array}{l}\text { SUCCESS-1 } \\
\text { (USA/Canada) }\end{array}$ & $\begin{array}{l}\text { Naproxen }(1000 \mathrm{mg} / \mathrm{d}) v \\
\text { celecoxib }(200,400 \mathrm{mg} / \mathrm{d})\end{array}$ & Osteoarthritis & 2736 & 12 & Yes & Yes & Yes & No & No & $\begin{array}{l}\text { Journal article, }{ }^{\text {w25 }} \text { study } \\
\text { register, }{ }^{\text {w26 }} \text { personal } \\
\text { communication }^{\text {w13 w14 }}\end{array}$ \\
\hline ADVANTAGE & $\begin{array}{l}\text { Naproxen }(1000 \mathrm{mg} / \mathrm{d}) v \\
\text { rofecoxib }(25 \mathrm{mg} / \mathrm{d})\end{array}$ & Osteoarthritis & 5557 & 14 & Yes & Unclear & Yes & Yes & No & $\begin{array}{l}\text { FDA reports, }{ }^{\text {w18 w27 journal }} \\
\text { article }^{\text {w28 } 29}\end{array}$ \\
\hline VIGOR & $\begin{array}{l}\text { Naproxen }(1000 \mathrm{mg} / \mathrm{d}) v \\
\text { rofecoxib }(50 \mathrm{mg} / \mathrm{d})\end{array}$ & $\begin{array}{l}\text { Rheumatoid } \\
\text { arthritis }\end{array}$ & 8076 & 54 & No & Yes & Yes & Yes & Yes & 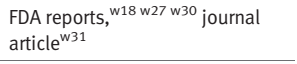 \\
\hline TARGET (0117) & $\begin{array}{l}\text { Naproxen }(1000 \mathrm{mg} / \mathrm{d}) v \\
\text { lumiracoxib }(400 \mathrm{mg} / \mathrm{d})\end{array}$ & Osteoarthritis & 9511 & 56 & Yes & Yes & Yes & Yes & No & $\begin{array}{l}\text { FDA reports, }{ }^{\text {w32 }} \text { journal article, }{ }^{\text {w33 }} \\
\text { personal communication } 6 \text { w7 }\end{array}$ \\
\hline $\begin{array}{l}\text { CLASS (N49- } \\
98-02-035)\end{array}$ & $\begin{array}{l}\text { Ibuprofen }(2400 \mathrm{mg} / \mathrm{d}) v \\
\text { celecoxib }(800 \mathrm{mg} / \mathrm{d})\end{array}$ & $\begin{array}{l}\text { Osteoarthritis and } \\
\text { rheumatoid arthritis }\end{array}$ & 3975 & 65 & Yes & Yes & Yes & No & No & 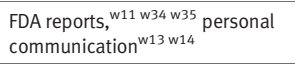 \\
\hline TARGET (2332) & $\begin{array}{l}\text { Ibuprofen }(2400 \mathrm{mg} / \mathrm{d}) v \\
\text { lumiracoxib }(400 \mathrm{mg} / \mathrm{d})\end{array}$ & Osteoarthritis & 8814 & 56 & Yes & Yes & Yes & Yes & No & $\begin{array}{l}\text { FDA reports, }{ }^{\mathrm{w} 23} \text { journal article, }{ }^{\mathrm{w} 33} \\
\text { personal communication }{ }^{\mathrm{w} 6 \mathrm{w7}}\end{array}$ \\
\hline CAESAR & $\begin{array}{l}\text { Diclofenac }(100 \mathrm{mg} / \mathrm{d}) v \\
\text { celecoxib }(200 \mathrm{mg} / \mathrm{d})\end{array}$ & Osteoarthritis & 916 & 52 & Yes & Yes & Yes & No & No & $\begin{array}{l}\text { FDA reports, }{ }^{\text {w11 }} \text { study register, }{ }^{\text {w36 }} \\
\text { conference abstract, }{ }^{\text {w37 }} \text { personal } \\
\text { communication }{ }^{\text {w13 w14 }}\end{array}$ \\
\hline $\begin{array}{l}\text { CLASS (N49- } \\
\text { 98-02-102) }\end{array}$ & $\begin{array}{l}\text { Diclofenac }(150 \mathrm{mg} / \mathrm{d}) v \\
\text { celecoxib }(800 \mathrm{mg} / \mathrm{d})\end{array}$ & $\begin{array}{l}\text { Osteoarthritis and } \\
\text { rheumatoid arthritis }\end{array}$ & 3993 & 52 & Yes & Yes & Yes & No & No & $\begin{array}{l}\text { FDA reports, }{ }^{\text {w11 w34 w35 }} \text { personal } \\
\text { communication }{ }^{\text {w13 w14 }}\end{array}$ \\
\hline Emery 1999 & $\begin{array}{l}\text { Diclofenac }(150 \mathrm{mg} / \mathrm{d}) v \\
\text { celecoxib }(400 \mathrm{mg} / \mathrm{d})\end{array}$ & $\begin{array}{l}\text { Rheumatoid } \\
\text { arthritis }\end{array}$ & 655 & 24 & No & Yes & Yes & No & No & $\begin{array}{l}\text { Journal article, }{ }^{\text {w38 }} \text { personal } \\
\text { communication }^{\text {w13 w14 }}\end{array}$ \\
\hline $\begin{array}{l}\text { SUCCESS-1 } \\
\text { (World) }\end{array}$ & $\begin{array}{l}\text { Diclofenac }(100 \mathrm{mg} / \mathrm{d}) v \\
\text { celecoxib }(200,400 \mathrm{mg} / \mathrm{d})\end{array}$ & Osteoarthritis & 10458 & 12 & Yes & Yes & Yes & No & No & $\begin{array}{l}\text { Journal article, }{ }^{\text {w25 }} \text { study } \\
\text { register, }{ }^{\text {w26 }} \text { personal } \\
\text { communication }^{\text {w13 w14 }}\end{array}$ \\
\hline EDGE & $\begin{array}{l}\text { Diclofenac }(150 \mathrm{mg} / \mathrm{d}) v \\
\text { etoricoxib }(90 \mathrm{mg} / \mathrm{d})\end{array}$ & Osteoarthritis & 7111 & 73 & Yes & Yes & Yes & Yes & No & FDA reports, ${ }^{\text {w39 }}$ journal article ${ }^{\text {w40 }}$ \\
\hline EDGE ॥ & $\begin{array}{l}\text { Diclofenac }(150 \mathrm{mg} / \mathrm{d}) v \\
\text { etoricoxib }(90 \mathrm{mg} / \mathrm{d})\end{array}$ & $\begin{array}{l}\text { Rheumatoid } \\
\text { arthritis }\end{array}$ & 4086 & 150 & Yes & Yes & Yes & Yes & No & Journal article ${ }^{\mathrm{w} 41}$ \\
\hline MEDAL & $\begin{array}{l}\text { Diclofenac }(150 \mathrm{mg} / \mathrm{d}) v \\
\text { etoricoxib }(60,90 \mathrm{mg} / \mathrm{d})\end{array}$ & $\begin{array}{l}\text { Osteoarthritis and } \\
\text { rheumatoid arthritis }\end{array}$ & 23498 & 176 & Yes & Yes & Yes & Yes & No & Journal article $e^{w 42}$ \\
\hline Cannon 2000 & $\begin{array}{l}\text { Diclofenac }(150 \mathrm{mg} / \mathrm{d}) v \text { rofecoxib } \\
(12.5,25 \mathrm{mg} / \mathrm{d})\end{array}$ & Osteoarthritis & 784 & 52 & No & Yes & Yes & No & No & 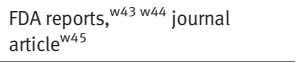 \\
\hline Saag 2000 & $\begin{array}{l}\text { Diclofenac }(150 \mathrm{mg} / \mathrm{d}) v \text { rofecoxib } \\
(12.5,25 \mathrm{mg} / \mathrm{d})\end{array}$ & Osteoarthritis & 693 & 54 & No & Yes & Yes & No & No & $\begin{array}{l}\text { FDA reports, }{ }^{\text {w3 }} \text { w44 journal } \\
\text { article }^{\text {w46 }}\end{array}$ \\
\hline $\begin{array}{l}\text { Fleischmann } \\
2003^{\star \star}\end{array}$ & $\begin{array}{l}\text { Celecoxib }(200 \mathrm{mg} / \mathrm{d}) v \\
\text { lumiracoxib }(200,400 \mathrm{mg} / \mathrm{d})\end{array}$ & Osteoarthritis & 1376 & 13 & Yes & Yes & Yes & No & No & $\begin{array}{l}\text { Journal article, }{ }^{\mathrm{w} 47} \text { personal } \\
\text { communication }^{\mathrm{w} 6 \mathrm{w7}}\end{array}$ \\
\hline $\begin{array}{l}\text { Tannenbaum } \\
2004^{\star \star}\end{array}$ & $\begin{array}{l}\text { Celecoxib }(200 \mathrm{mg} / \mathrm{d}) v \\
\text { lumiracoxib }(200,400 \mathrm{mg} / \mathrm{d})\end{array}$ & Osteoarthritis & 1459 & 13 & Yes & Yes & Yes & No & Yes & $\begin{array}{l}\text { Journal article, }{ }^{\text {w48 }} \text { personal } \\
\text { communication }^{\text {w6 }} \mathrm{w}^{2}\end{array}$ \\
\hline
\end{tabular}

FDA=US Food and Drug Administration. See web extra appendix 3 for full names of trials and references to sources.

*Number of randomised patients of included trial arms.

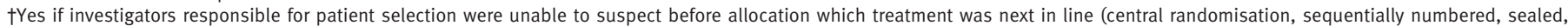
opaque assignment envelopes, coded drug packs).

†Yes if drugs looked similar (for example, matching placebo) or double dummy was used.

\$Relates to myocardial infarctions only. See web extra appendix 2 for more detailed description of external adjudication of events in individual trials.

TYes if all randomised patients were included in analysis.

**Placebo arm excluded ((100 patient years of follow-up). 
Table 2 |Number of events by randomised intervention for each outcome

\begin{tabular}{|c|c|c|c|c|c|c|c|}
\hline Trial & Intervention & $\begin{array}{l}\text { Patient } \\
\text { years* }\end{array}$ & $\begin{array}{l}\text { Myocardial } \\
\text { infarction } †\end{array}$ & Stroke† & $\begin{array}{c}\text { Cardiovascular } \\
\text { death }\end{array}$ & Death $\ddagger$ & $\begin{array}{l}\text { APTC composite } \\
\text { outcome }\end{array}$ \\
\hline ADAPT & Placebo & 1982 & 13 & 7 & 3 & 12 & 22 \\
\hline ADAPT & Naproxen & 1332 & 13 & 10 & 3 & 11 & 23 \\
\hline ADAPT & Celecoxib & 1346 & 8 & 7 & 4 & 9 & 17 \\
\hline Aisen 2003 & Placebo & 115 & 1 & 1 & 1 & 1 & 2 \\
\hline Aisen 2003 & Naproxen & 124 & 0 & 5 & 1 & 1 & 6 \\
\hline Aisen 2003 & Rofecoxib & 126 & 3 & 4 & 2 & 2 & 7 \\
\hline Geusens 2004 & Placebo & 111 & 0 & 1 & 0 & 0 & 1 \\
\hline Geusens 2004 & Naproxen & 118 & 0 & 0 & 0 & 0 & 0 \\
\hline Geusens 2004 & Lumiracoxib & 234 & 2 & 3 & 2 & 2 & 6 \\
\hline APC & Placebo & 1558 & 3 & 3 & 1 & 6 & 7 \\
\hline APC & Celecoxib & 3124 & 18 & 8 & 11 & 18 & 37 \\
\hline GAIT & Placebo & 140 & NA & NA & 0 & 0 & NA \\
\hline GAIT & Celecoxib & 146 & NA & NA & 0 & 0 & NA \\
\hline IQ5-97-02-001 & Placebo & 120 & 0 & 3 & 2 & 4 & 3 \\
\hline IQ5-97-02-001 & Celecoxib & 285 & 2 & 7 & 8 & 13 & 12 \\
\hline PreSAP & Placebo & 1570 & 4 & 7 & 4 & 7 & 12 \\
\hline PreSAP & Celecoxib & 2331 & 9 & 9 & 4 & 11 & 21 \\
\hline Lehmann 2005 & Placebo & 98 & 1 & 0 & 0 & 0 & 1 \\
\hline Lehmann 2005 & Celecoxib & 99 & 0 & 0 & 0 & 0 & 0 \\
\hline Lehmann 2005 & Lumiracoxib & 200 & 0 & 1 & 1 & 1 & 2 \\
\hline APPROVe & Placebo & 5711 & 18 & 9 & 13 & 28 & 34 \\
\hline APPROVe & Rofecoxib & 5658 & 34 & 19 & 16 & 36 & 59 \\
\hline Reines 2004 & Placebo & 293 & 4 & 5 & 3 & 8 & 12 \\
\hline Reines 2004 & Rofecoxib & 273 & 2 & 1 & 4 & 14 & 4 \\
\hline Thal 2005 & Placebo & 1820 & 13 & 15 & 3 & 20 & 28 \\
\hline Thal 2005 & Rofecoxib & 1599 & 22 & 7 & 13 & 41 & 33 \\
\hline VICTOR & Placebo & 986 & 1 & 3 & 3 & NA & 6 \\
\hline VICTOR & Rofecoxib & 928 & 6 & 3 & 4 & NA & 9 \\
\hline ViP & Placebo & 1102 & 5 & 3 & 3 & 4 & 9 \\
\hline ViP & Rofecoxib & 1099 & 6 & 2 & 1 & 3 & 8 \\
\hline A3191152 & Naproxen & 130 & 0 & 0 & 0 & 0 & 0 \\
\hline A3191152 & Celecoxib & 131 & 0 & 0 & 0 & 0 & 0 \\
\hline SUCCESS-1 (USA/Canada) & Naproxen & 165 & 1 & 2 & 0 & 0 & 3 \\
\hline SUCCESS-1 (USA/Canada) & Celecoxib & 353 & 4 & 2 & 1 & 1 & 4 \\
\hline ADVANTAGE & Naproxen & 526 & 1 & 6 & 0 & 4 & 7 \\
\hline ADVANTAGE & Rofecoxib & 528 & 5 & 1 & 4 & 5 & 10 \\
\hline VIGOR & Naproxen & 2008 & 4 & 9 & 7 & 15 & 17 \\
\hline VIGOR & Rofecoxib & 2007 & 20 & 11 & 9 & 22 & 34 \\
\hline TARGET (0117) & Naproxen & 4156 & 7 & 13 & 8 & 11 & 27 \\
\hline TARGET (0117) & Lumiracoxib & 4197 & 15 & 17 & 11 & 14 & 40 \\
\hline CLASS (N49-98-02-035) & Ibuprofen & 1123 & 9 & 6 & 6 & 8 & 17 \\
\hline CLASS (N49-98-02-035) & Celecoxib & 1184 & 9 & 2 & 6 & 9 & 13 \\
\hline TARGET (2332) & Ibuprofen & 3709 & 5 & 9 & 10 & 15 & 23 \\
\hline TARGET (2332) & Lumiracoxib & 3795 & 5 & 8 & 8 & 13 & 19 \\
\hline CAESAR & Diclofenac & 432 & 5 & 5 & 4 & 5 & 12 \\
\hline CAESAR & Celecoxib & 415 & 4 & 1 & 5 & 6 & 8 \\
\hline CLASS (N49-98-02-102) & Diclofenac & 1081 & 5 & 6 & 7 & 9 & 16 \\
\hline CLASS (N49-98-02-102) & Celecoxib & 1136 & 10 & 2 & 6 & 10 & 17 \\
\hline Emery 1999 & Diclofenac & 125 & 0 & 0 & 0 & 0 & 0 \\
\hline Emery 1999 & Celecoxib & 133 & 1 & 0 & 0 & 1 & 1 \\
\hline SUCCESS-1 (World) & Diclofenac & 745 & 0 & 4 & 4 & 5 & 4 \\
\hline SUCCESS-1 (World) & Celecoxib & 1472 & 6 & 6 & 1 & 4 & 12 \\
\hline EDGE & Diclofenac & 2607 & 11 & 6 & 4 & 8 & 12 \\
\hline EDGE & Etoricoxib & 2789 & 19 & 4 & 5 & 10 & 25 \\
\hline EDGE II & Diclofenac & 3251 & 25 & 12 & 7 & 18 & 42 \\
\hline
\end{tabular}




\begin{tabular}{|c|c|c|c|c|c|c|c|}
\hline Trial & Intervention & $\begin{array}{l}\text { Patient } \\
\text { years* }\end{array}$ & $\begin{array}{l}\text { Myocardial } \\
\text { infarction } †\end{array}$ & Stroke $†$ & $\begin{array}{c}\text { Cardiovascular } \\
\text { death }\end{array}$ & Death $\ddagger$ & $\begin{array}{l}\text { APTC composite } \\
\text { outcome }\end{array}$ \\
\hline EDGE II & Etoricoxib & 3266 & 14 & 9 & 7 & 24 & 30 \\
\hline MEDAL & Diclofenac & 19103 & 88 & 42 & 38 & 105 & 149 \\
\hline MEDAL & Etoricoxib & 19970 & 84 & 48 & 39 & 95 & 154 \\
\hline Cannon 2000 & Diclofenac & 256 & 1 & 1 & 2 & 2 & 4 \\
\hline Cannon 2000 & Rofecoxib & 494 & 2 & 0 & 1 & 1 & 3 \\
\hline Saag 2000 & Diclofenac & 219 & 1 & 1 & 2 & 4 & 2 \\
\hline Saag 2000 & Rofecoxib & 443 & 2 & 1 & 0 & 0 & 3 \\
\hline Fleischmann 2003 & Celecoxib & 99 & 0 & 0 & 0 & 0 & 0 \\
\hline Fleischmann 2003 & Lumiracoxib & 207 & 2 & 0 & 0 & 0 & 2 \\
\hline Tannenbaum 2004 & Celecoxib & 110 & 0 & 0 & 0 & 0 & NA \\
\hline Tannenbaum 2004 & Lumiracoxib & 225 & 1 & 0 & 0 & 0 & NA \\
\hline Overall & & 117218 & 554 & 377 & 312 & 676 & 1091 \\
\hline
\end{tabular}

APTC=Antiplatelet Trialists' Collaboration; NA=not available. See web extra appendix 3 for full names of trials.

*Slightly different numbers of patient years might be used for analysis of each outcome but only number of patient years for primary outcome

"incidence of myocardial infarction" is provided here.

†Includes fatal and non-fatal events.

$\ddagger$ Death from any cause.

\section{Antiplatelet Trialists' Collaboration composite outcome}

Thirty trials with 1091 accumulated events contributed to the analysis on the Antiplatelet Trialists' Collaboration composite outcome (table 2). All drugs seemed to be associated with increased risks of the composite of non-fatal myocardial infarction, non-fatal stroke, or cardiovascular death compared with placebo (fig 2). The estimated rate ratios were greater than 1.3 for ibuprofen $(2.26,1.11$ to 4.89$)$, diclofenac $(1.60,0.85$ to $2.99)$, celecoxib $(1.43,0.94$ to 2.16$)$, etoricoxib (1.53, 0.74 to 3.17$)$, rofecoxib $(1.44,1.00$ to 1.99$)$, and lumiracoxib $(2.04,1.13$ to 4.24$)$. Figure 3 presents an overview of pairwise comparisons (rate ratios with 95\% credibility intervals) of all drugs on all outcomes.

\section{Posterior probabilities}

Figure 4 presents posterior probability curves with resulting confidence levels for the different drugs compared with placebo and different outcomes. For example, the probability that rofecoxib increases the risk of myocardial infarction by less than $30 \%$ is $3 \%$ or conversely there is $97 \%$ confidence that rofecoxib increases the risk by at least $30 \%$ (corresponding to a rate ratio of 1.3). The curves can also be used to examine the overall pattern of available evidence of a specific drug. For example, the relatively steep increases in all but one of the posterior probability curves for naproxen points to the robust evidence available for naproxen. In contrast, the relatively flat curves for etoricoxib indicate a relative lack of available evidence. However, the mostly large effects seen for etoricoxib (indicated by the right shift of the curves) nevertheless allow conclusions for clinically relevant risk increases.

Evaluation of models, variation, and sensitivity analyses The model fit was good for all outcomes (see web extra appendix 2). Estimates of statistical heterogeneity between direct comparisons were generally low, except for myocardial infarction (range of $\tau^{2}$ across outcomes: 0.03 to 0.12 ; see web extra appendix 2 ). Inconsistency between direct and indirect comparisons was low for all outcomes (range of median inconsistency factors $2 \%$ to $29 \%$; see web extra appendix 2). However, given the relatively low number of trials and events, relevant heterogeneity or inconsistency between trials could not be ruled out. Detailed results of the sensitivity analyses are presented in web extra appendix 2: results were all compatible with main analyses. Many of the estimates were imprecise, however, and do not allow for firm conclusions to be drawn.

\section{DISCUSSION}

In this network meta-analysis of cardiovascular safety data of seven non-steroidal anti-inflammatory drugs and placebo, naproxen seemed least harmful (fig 3). Safety profiles of individual drugs varied considerably depending on the outcome, and estimated rate ratios for comparisons with placebo were generally imprecise. Non-steroidal anti-inflammatory drugs are mainly used for symptomatic treatment of musculoskeletal conditions. Clearly, as for any symptomatic treatment, doing more harm than good with this class of drugs should be avoided (primum non nocere). Taking this into account, we presented confidence levels (fig 4), which can be interpreted as confidence that a drug is associated with an increase in risk that is smaller than a specified threshold. For the primary outcome of myocardial infarction, the confidence that the increase in risk associated with the evaluated non-steroidal antiinflammatory drugs does not exceed 30\% (the risk increase used as non-inferiority margin in the Multinational Etoricoxib and Diclofenac Arthritis Long-term programme ${ }^{16}$ ) is sufficiently high only for naproxen. Conversely, we are confident that several other drugs -ibuprofen, diclofenac, etoricoxib, and lumiracoxib - are associated with a risk increase of more than $30 \%$ on several cardiovascular outcomes.

Although our analysis covered more than 100000 patient years of follow-up, the number of events for most outcomes was low and our estimates of rate ratios imprecise, as indicated by wide credibility intervals. Given the low event rates in the included trials, 
establishing the cardiovascular safety of a preparation with sufficient precision would require a trial with more than 100000 patients followed up for at least one year. Such a large trial may be difficult to carry out, considering the limited amount of funding available and the inherent ethical problems. Although estimated rate ratios indicated harmful effects in most drugs on the majority of outcomes, conventional levels of statistical significance were reached in about 30\% of comparisons with placebo. Absence of statistically robust evidence of a harmful effect should not be confused with evidence of absence of cardiovascular
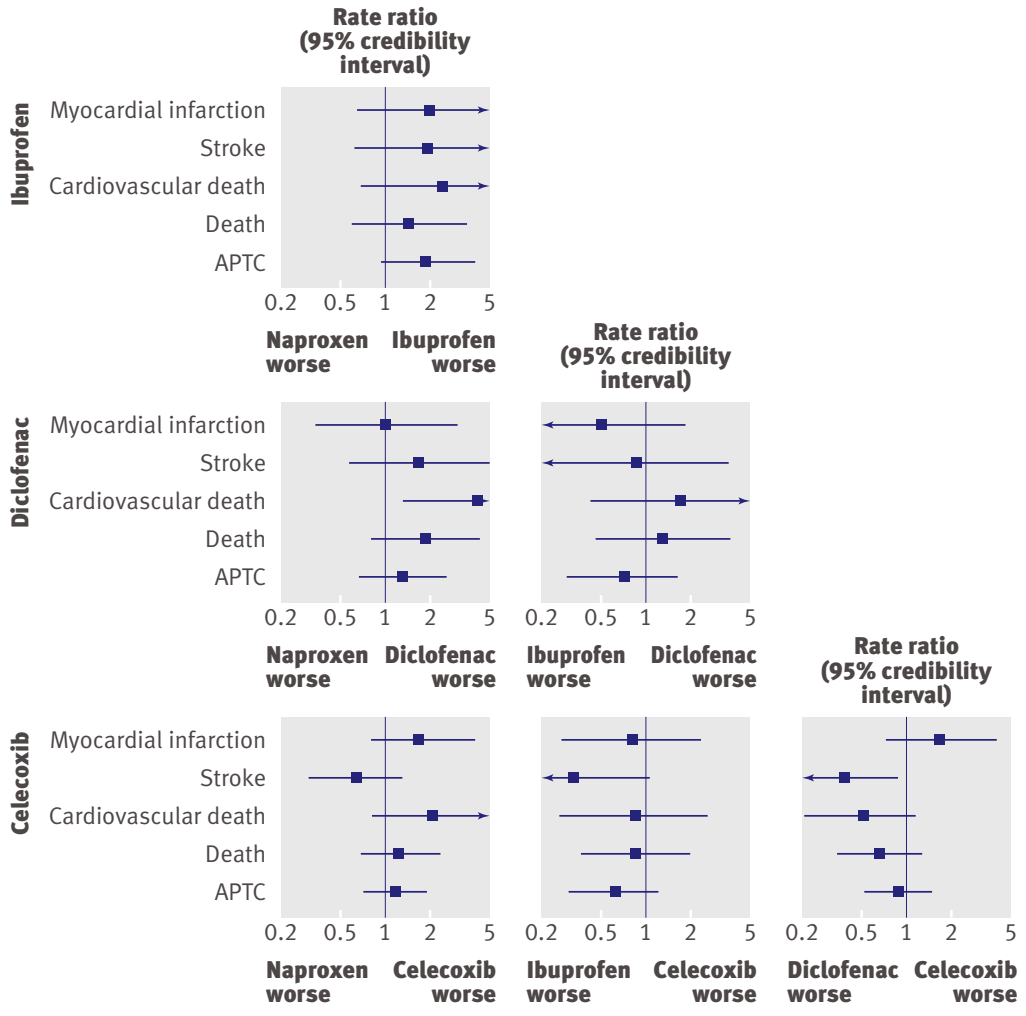

$\begin{array}{rr}\text { 읓 } & \text { Myocardial infarction } \\ \text { 는 } & \text { Stroke } \\ \text { 는 } & \text { Cardiovascular death }\end{array}$
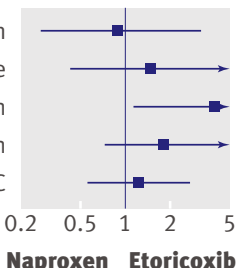

Naproxen Etoricoxib
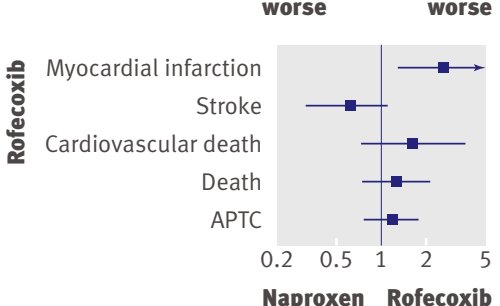

worse worse

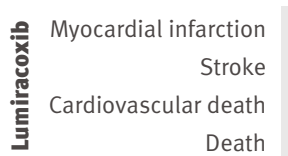

Death
APTC
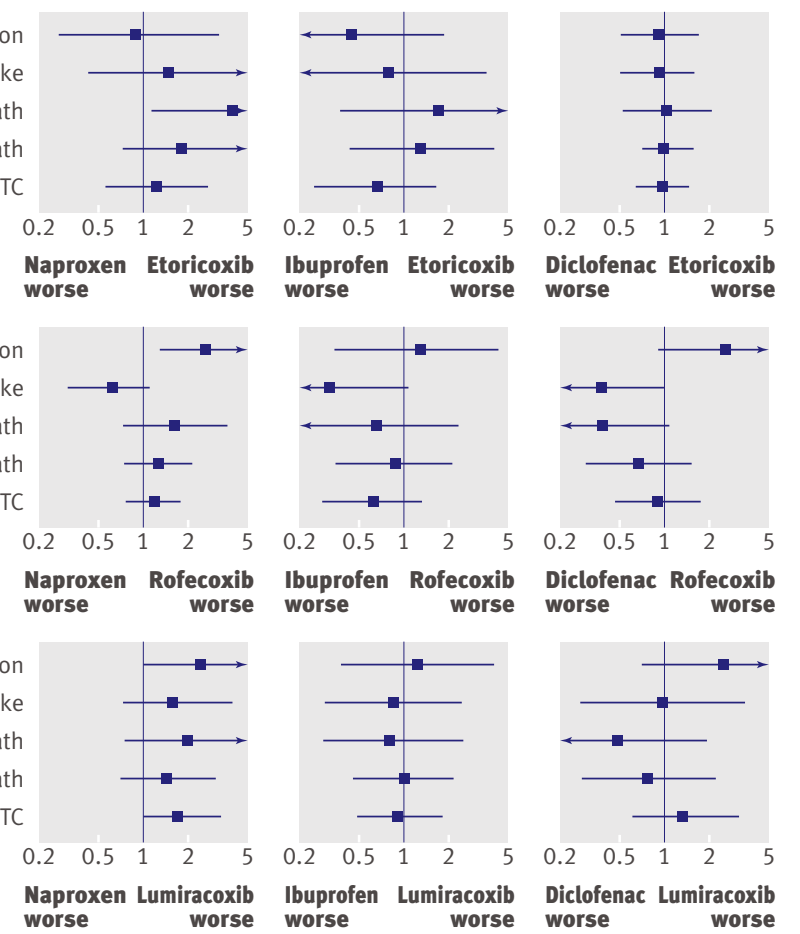

worse
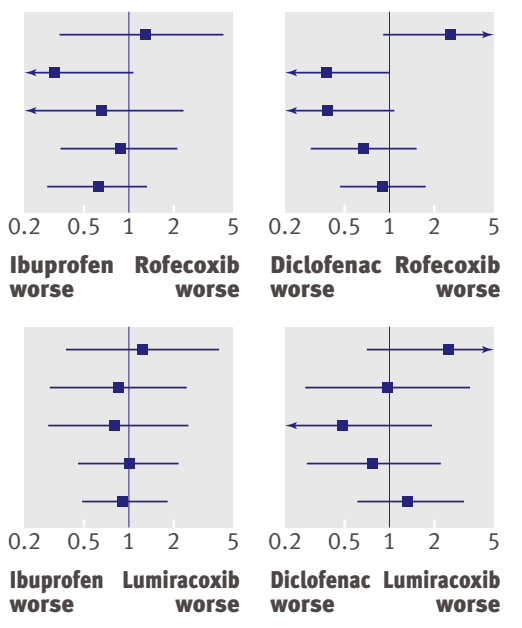

Ibuprofen

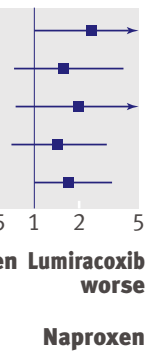

worse worse worse

Diclofenac
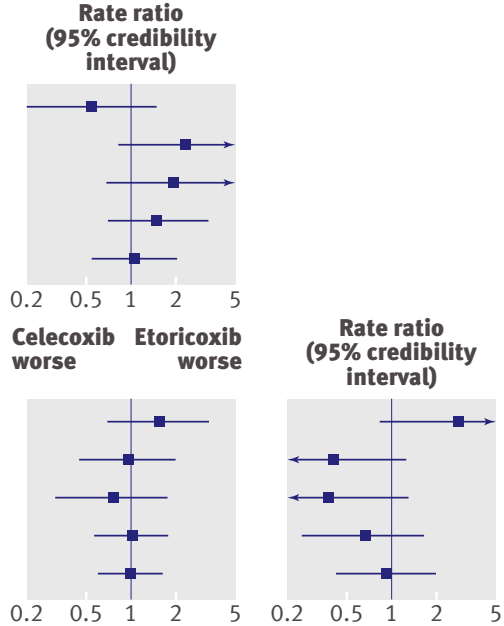

Celecoxib Rofecoxib worse

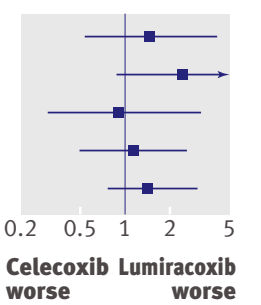

Etorice
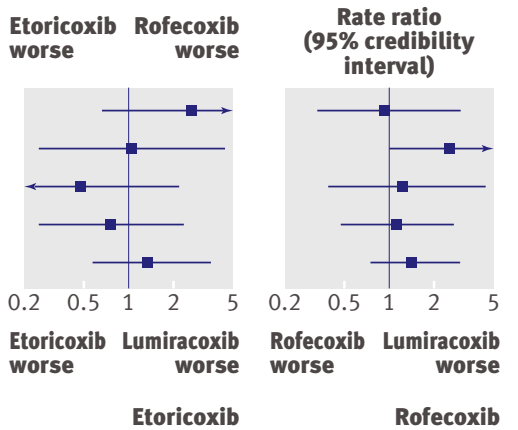

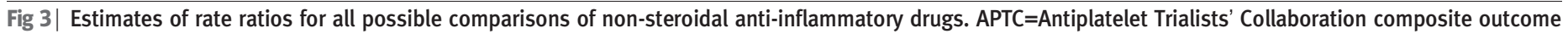



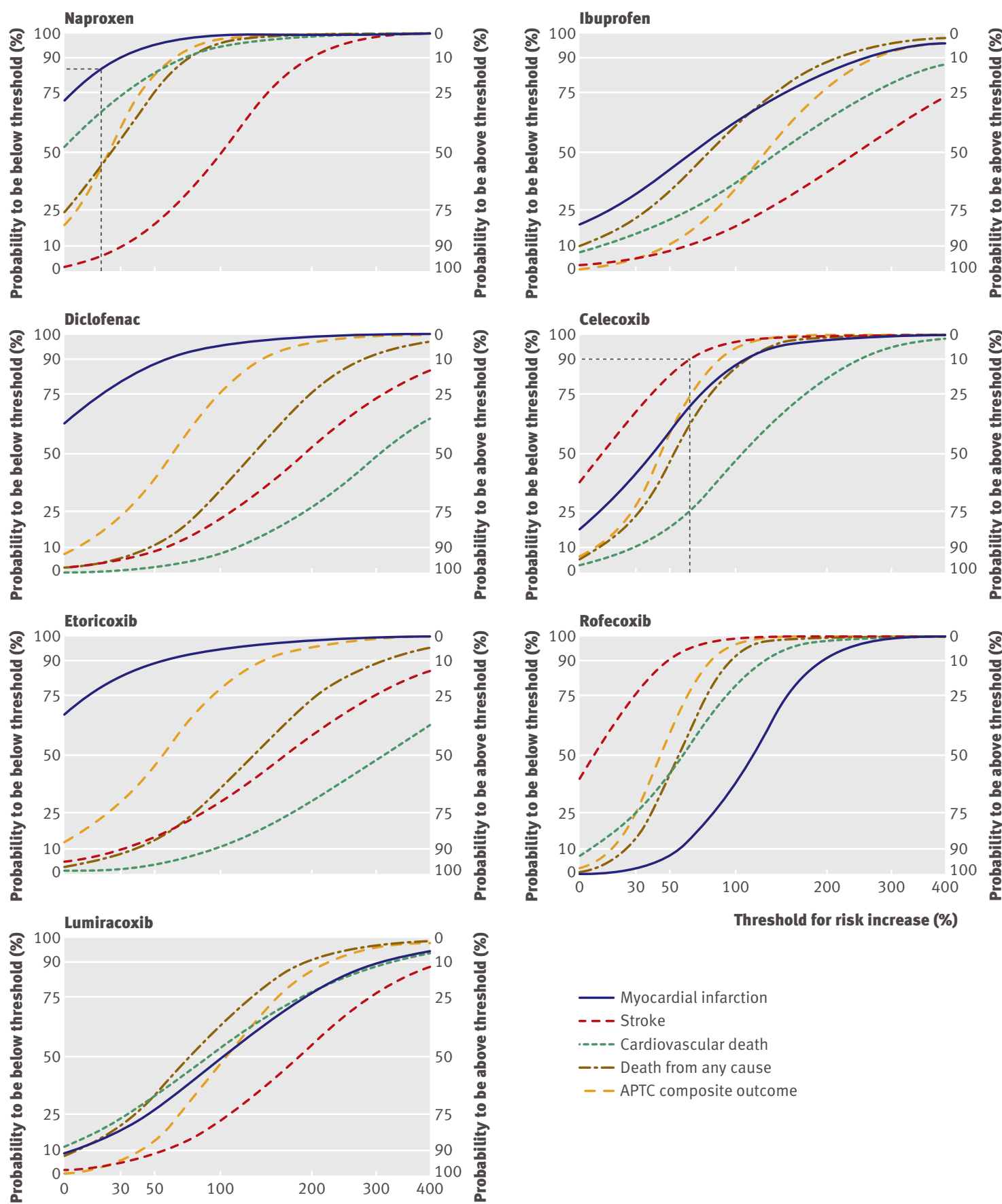

Threshold for risk increase (\%)

Threshold for risk increase (\%)

Fig 4 | Posterior probabilities for specified rate ratios. Curves can be used to extract a probability corresponding to a specified minimally clinically relevant rate ratio or to extract a minimal rate ratio corresponding to a specified probability or confidence level. For example, an increase in risk for myocardial infarction of at least $20 \%$ (rate ratio of at least 1.2 ) may be considered clinically relevant. The curve for naproxen indicates that the probability of the drug being associated with a rate ratio below this threshold is about $83 \%$. Conversely, a probability of $90 \%$ may be considered as appropriate evidence for the outcome stroke. The curve for celecoxib indicates that someone can be $90 \%$ confident that celecoxib increases the risk for stroke by no more than $65 \%$ (rate ratio of 1.65). APTC=Antiplatelet Trialists' Collaboration

toxicity for the evaluated drugs. ${ }^{18}$ Posterior probabilities may increase our understanding of cardiovascular safety data in this situation and allow for different notions about what constitutes a clinically relevant increase in risk. Most will agree that a rate ratio of 1.3 indicates a clinically relevant increase in risk as was used as the upper bound of the non-inferiority margin by studies in the Multinational Etoricoxib and Diclofenac Arthritis Long-term programme, ${ }^{16}$ and we are confident that several drugs are associated with a risk increase higher than this margin for several outcomes. 
Some may argue that absolute rates of events were low and clinically irrelevant, despite increases in rate ratios. Event rates in the included trials are considerably lower than in routine clinical settings. ${ }^{4}$ Numbers needed to harm are therefore lower in routine settings than in most trial populations. The estimated rate ratios observed in our study will translate into clinically relevant numbers needed to harm in most routine populations of patients taking non-steroidal antiinflammatory drugs, who are typically at moderate to high risks for cardiovascular events. ${ }^{19}$

\section{Strengths and weaknesses of the meta-analysis}

Our analysis has several limitations. Firstly, we were unable to consider all non-steroidal anti-inflammatory drugs in our analysis: large scale randomised controlled trials are lacking for most of the older drugs and even for some newer ones, such as valdecoxib or meloxicam. Nevertheless, we were able to include all relevant cyclo-oxygenase- 2 inhibitors, except valdecoxib, and the three most commonly used traditional non-steroidal anti-inflammatory drugs. ${ }^{2021}$ By disregarding small studies we minimised the risk of small study effects. ${ }^{22}$ Small studies would have had minimal impact on the analysis anyway, because of low numbers of events. ${ }^{23}$ Secondly, we were able to obtain unpublished data only for the trials of celecoxib and lumiracoxib, whereas Merck, the manufacturer of rofecoxib and etoricoxib, was not willing to provide unpublished safety data. Therefore some data were missing for trials sponsored by Merck. This is disconcerting in the light of the safety concerns raised by our analysis for both drugs, rofecoxib and etoricoxib, manufactured by Merck. Thirdly, the quality of our analysis is limited by the quality of the underlying data. Although the methodological quality of included trials was generally satisfactory, the quality of reporting was often less than optimal ${ }^{24}$ and we found discrepancies in the reported number of events between different sources of information for major trials including ADVANTAGE (Assessment of Differences between Vioxx and Naproxen To Ascertain Gastrointestinal Tolerability and Effectiveness), VIGOR (Vioxx Gastrointestinal Outcomes Research), and APPROVe (Adenomatous Polyp Prevention on Vioxx). ${ }^{325-27} \mathrm{Sev}$ eral trials lacked independent adjudication of events, therefore bias in either direction cannot be excluded, including bias towards the null owing to non-differential misclassification of events or assessor bias in trials without independent adjudication. ${ }^{43}$ Nevertheless, the analysis restricted to trials with independent adjudication of events supported the robustness of our main analysis (see web extra appendix 2). Fourthly, one study explored the effects of dosage and regimen in a pooled analysis of six randomised placebo controlled trials of celecoxib and found that lower dosages and once daily regimens that avoided continuous interference of the drug with prostaglandin metabolism were associated with lower relative risks for the cardiovascular composite outcome than higher dosages and twice daily regimens. ${ }^{28}$ We were unable to satisfactorily deal with these matters in our analysis mainly because of the complexity of the network and the low number of patients treated with low dosages. In addition, regimens used in clinical practice might differ from the regimens used in the included clinical trials. Intermittent usage seems to be more common in clinical practice than the chronic long term usage in the trials, resulting in less intense drug use. ${ }^{29}$ Because none of the trials used intermittent regimens and drugs were used for at least one year in most of the trials we were unable to investigate the impact of drug use on cardiovascular outcomes. Data from the General Practice Research Database indicate, however, that about half of the patients have patterns of drug intake comparable to those evaluated in the trials included in this network meta-analysis, ${ }^{29}$ and we submit that the results of our study are applicable to these patients. Finally, we carried out several sensitivity analyses to determine the robustness of the results. Unfortunately, owing to the low number of accumulated events, estimates from these analyses were imprecise and do not allow any meaningful conclusion. This is particularly true for the analysis restricted to patients with musculoskeletal conditions: many of the point estimates could not be derived at all and others seemed to contradict the main results, but credibility intervals were compatible with both, major benefits or detrimental harms.

We used a comprehensive search strategy and searched pertinent sources to retrieve potentially eligible randomised controlled trials. ${ }^{30}$ It therefore seems unlikely that we missed any relevant trial. Using network meta-analysis we were able to integrate all available randomised evidence on the cardiovascular safety of non-steroidal anti-inflammatory drugs in one analysis while fully preserving randomisation. ${ }^{31}$ The integration of direct and indirect comparisons results in a gain of statistical precision compared with previous analyses $^{423}$ and allows for formal comparisons of non-steroidal anti-inflammatory drugs with placebo. In the most comprehensive analysis to date, one study compared five cyclo-oxygenase- 2 selective inhibitors with placebo using conventional techniques. ${ }^{23}$ They found all estimates of relative risks of cardiovascular death imprecise and largely overlapping the null effect line, compatible with substantial harms or benefits. In contrast, our estimates were more precise compared with that study, which estimated a nearly identical rate ratio for the comparison of etoricoxib and placebo for the outcome cardiovascular death (rate ratio $4.4 v 4.07$ in our analysis). However, the confidence interval in that meta-analysis ranged from 0.2 to 119 whereas our credibility interval ranged from 1.23 to 15.7 , providing stronger evidence for increases in the risk of cardiovascular death. Also, wide confidence intervals around estimates for lumiracoxib did not allow for a conclusion on any of the outcomes in their analysis. In contrast, our analysis provided smaller intervals, and posterior probabilities indicated a high probability that lumiracoxib is associated with a clinically relevant increase in risk of cardiovascular outcomes. 


\section{WHAT IS ALREADY KNOWN ON THIS TOPIC}

Non-steroidal anti-inflammatory drugs (NSAIDs) have been the cornerstone of managing pain from osteoarthritis and other conditions

Debate surrounds the cardiovascular safety of cyclo-oxygenase-2 selective inhibitors, with similar concerns about traditional NSAIDs, leading to non-approval and withdrawal of newer preparations

Several meta-analyses were unable to resolve the debate because they failed to integrate all available randomised evidence in one analysis

\section{WHAT THIS STUDY ADDS}

Among the seven NSAIDs analysed, naproxen seemed least harmful for cardiovascular safety Safety profiles of individual drugs varied considerably depending on the outcome

The estimated rate ratios for comparisons with placebo were generally imprecise

\section{Comparison with other studies}

Our study confirms previous notions of regulatory bodies, mainly based on observational evidence, that all non-steroidal anti-inflammatory drugs are associated with an increased risk of cardiovascular adverse effects. $^{3233}$ Observational evidence is likely to be affected by confounding by indication. ${ }^{34}$ Our results are based on randomised evidence and we therefore believe that our study provides the best available evidence on the safety of this class of drugs. Nevertheless, our results are mostly compatible with the results of a meta-analysis of observational studies-for example, for naproxen, diclofenac, or rofecoxib-although some differences exist, especially for ibuprofen. ${ }^{5}$ Besides confounding by indication, these differences might be explained not only by differences in drug use between trials and observational studies ${ }^{29}$ but also by the quality of observational studies, which lead to high heterogeneity between studies and a possible underestimation of effects. ${ }^{535}$

We found no clear relation between specificity of cyclo-oxygenase-2 inhibitors and risk of cardiovascular events. This finding contrasts with previous claims that increased selectivity for cyclo-oxygenase2 inhibitors is associated with increased cardiovascular risk. ${ }^{36}$ Several mechanisms have been postulated, but the hypothesis of an imbalance between prostacyclin and thromboxane $\mathrm{A}_{2}$ leading to an increased risk for thombotic events gained most prominence. ${ }^{37}$ However, the lack of a clear association between specificity of cyclo-oxygenase- 2 inhibitors and cardiovascular risk implies that other mechanisms need to be considered. Multiple effects most probably contribute to the increased risk of cardiovascular events, including differential effects on prostacyclin and thromboxane $\mathrm{A}_{2}$ synthesis, ${ }^{37}$ endothelial function and nitric oxide production, ${ }^{38}$ blood pressure,${ }^{39}$ volume retention and other renal effects. ${ }^{40}$ In addition, differences in pharmacokinetics may contribute to the toxicity profile ${ }^{41}$; drugs with a long half life prescribed once daily (such as rofecoxib) and drugs with a shorter half life prescribed more than once daily (such as diclofenac) may be more likely to continuously interfere with the cyclo- oxygenase system than drugs with a shorter half life prescribed once daily (such as celecoxib). ${ }^{28}$

\section{Implications and conclusions}

The observation that cardiovascular risk is not clearly associated with specificity of cyclo-oxygenase- 2 inhibitors implies that no prediction of cardiovascular risk can be made based on such specificity. Therefore the use of other non-steroidal anti-inflammatory drugs not covered by our analysis should be reconsidered, as well as the over the counter availability of non-steroidal anti-inflammatory drugs such as diclofenac or ibuprofen. In general, naproxen seems to be the safest analgesic for patients with osteoarthritis in cardiovascular terms but this advantage has to be weighed against gastrointestinal toxicity and the need for concomitant prescription of a proton pump inhibitor in many patients. In the light of the results of one study, ${ }^{28}$ celecoxib $400 \mathrm{mg}$ prescribed once daily may be considered as an alternative option. Other alternatives include paracetamol and opioids. Compared with placebo, however, paracetamol results in only a small reduction in pain and may be associated with clinically relevant hepatotoxicity, even in dosages recommended for musculoskeletal pain. ${ }^{42}$ The analgesic effect of opioids is somewhat more pronounced but outweighed by large increases in the risk of adverse events. ${ }^{44}$ In conclusion, the options for the treatment of chronic musculoskeletal pain are limited and patients and clinicians need to be aware that cardiovascular risk needs to be taken into account when prescribing.

We thank all independent trialists, Novartis, and Pfizer for providing unpublished data and Malcom Sturdy for development of the database. Contributors: ST and SR contributed equally to this work. ST, SR, SW, and PJ had full access to all of the data in the study and take responsibility for the integrity of the data and the accuracy of the data analysis. PJ conceived and designed the study. ST, SR, and BT acquired the data. Al authors analysed and interpreted the data. ST, SR, and PJ drafted the manuscript. SW, PH, BT, PMV, and ME critically revised the manuscript for important intellectual content. ST, SW, and PJ carried out the statistical analysis. SR, ME and PJ obtained funding. SR, PH, BT, PMV, ME, and PJ provided administrative support. PJ supervised the study. Funding: SR, ME, and P) received grants (Nos 4053-40-104762/3 and 3200-066378) from the Swiss National Science Foundation's national research programme 53 on musculoskeletal health. PJ was a programme for social medicine, preventive and epidemiological research senior research fellow funded by the Swiss National Science Foundation (grant No 3233-066377). CTU Bern is supported by the Swiss National Science Foundation. The Swiss National Science Foundation, had no role in the study design, data collection, data analysis, data interpretation, writing of the manuscript, or decision to submit the manuscript.

Competing interests: All authors have completed the Unified Competing Interest form at www.icmje.org/coi_disclosure.pdf (available on reques from the corresponding author) and declare: no support from any institution for the submitted work besides the funding as described above; no financial relationships with any institutions that might have an interest in the submitted work in the previous 3 years; no other relationships or activities that could appear to have influenced the submitted work.

Ethical approval: Not required.

Data sharing: No additional data available.

1 Dai C, Stafford RS, Alexander GC. National trends in cyclooxygenase 2 inhibitor use since market release: nonselective diffusion of a selectively cost-effective innovation. Arch Intern Med 2005;165:171-7. 
2 Kaufman DW, Kelly JP, Rosenberg L, Anderson TE, Mitchell AA. Recent patterns of medication use in the ambulatory adult population of the United States: the Slone survey. JAMA 2002;287:337-44.

3 Bresalier RS, Sandler RS, Quan H, Bolognese JA, Oxenius B, Horgan K, et al. Cardiovascular events associated with rofecoxib in a colorectal adenoma chemoprevention trial. N Engl J Med 2005;352:1092-102.

4 Juni P, Nartey L, Reichenbach S, Sterchi R, Dieppe PA, Egger M. Risk of cardiovascular events and rofecoxib: cumulative meta-analysis. Lancet 2004;364:2021-9.

5 McGettigan P, Henry D. Cardiovascular risk and inhibition of cyclooxygenase: a systematic review of the observational studies of selective and nonselective inhibitors of cyclooxygenase 2. JAMA 2006;296:1633-44.

6 Avorn J. Keeping science on top in drug evaluation. N Engl J Med 2007;357:633-5.

7 Borer JS. Cyclooxygenase inhibition: what should we do to resolve the confusion? An American perspective. J Cardiovasc Pharmacol 2006;47:S87-91.

8 Psaty BM, Lumley T, Furberg CD, Schellenbaum G, Pahor M Alderman $\mathrm{MH}$, et al. Health outcomes associated with various antihypertensive therapies used as first-line agents: a network metaanalysis. JAMA 2003;289:2534-44.

9 Cooper NJ, Sutton AJ, Lu G, Khunti K. Mixed comparison of stroke prevention treatments in individuals with nonrheumatic atrial fibrillation. Arch Intern Med 2006;166:1269-75.

10 Aisen PS, Schafer KA, Grundman M, Pfeiffer E, Sano M, Davis KL, et al. Effects of rofecoxib or naproxen vs placebo on Alzheimer disease progression: a randomized controlled trial. JAMA 2003;289:2819-26.

11 Collaborative overview of randomised trials of antiplatelet therapyI: prevention of death, myocardial infarction, and stroke by prolonged antiplatelet therapy in various categories of patients. Antiplatelet Trialists' Collaboration. BMJ 1994;308:81-106.

12 Sweeting MJ, Sutton AJ, Lambert PC. What to add to nothing? Use and avoidance of continuity corrections in meta-analysis of sparse data. Stat Med 2004;23:1351-75.

13 Higgins JP, Whitehead A. Borrowing strength from external trials in a meta-analysis. Stat Med 1996;15:2733-49.

14 Lu G, Ades AE. Combination of direct and indirect evidence in mixed treatment comparisons. Stat Med 2004;23:3105-24.

15 Shakespeare TP, Gebski VJ, Veness MJ, Simes J. Improving interpretation of clinical studies by use of confidence levels, clinical significance curves, and risk-benefit contours. Lancet 2001;357:1349-53.

16 Cannon CP, Curtis SP, FitzGerald GA, Krum H, Kaur A, Bolognese JA, et al. Cardiovascular outcomes with etoricoxib and diclofenac in patients with osteoarthritis and rheumatoid arthritis in the Multinational Etoricoxib and Diclofenac Arthritis Long-term (MEDAL) programme: a randomised comparison. Lancet 2006;368:1771-81.

17 Lu GB, Ades AE. Assessing evidence inconsistency in mixed treatment comparisons. J Am Stat Assoc 2006;101:447-59.

18 Altman DG, Bland JM. Absence of evidence is not evidence of absence. BM/ 1995;311:485.

19 Ray WA, Stein CM, Daugherty JR, Hall K, Arbogast PG, Griffin MR. COX 2 selective non-steroidal anti-inflammatory drugs and risk of serious coronary heart disease. Lancet 2002;360:1071-3.

20 Fries JF, Murtagh KN, Bennett M, Zatarain E, Lingala B, Bruce B. The rise and decline of nonsteroidal antiinflammatory drug-associated gastropathy in rheumatoid arthritis. Arthritis Rheum 2004; 50:2433-40

21 Paulose-Ram R, Hirsch R, Dillon C, Gu Q. Frequent monthly use of selected non-prescription and prescription non-narcotic analgesics among US adults. Pharmacoepidemiol Drug Saf 2005;14:257-66.

22 Egger M, Davey Smith G, Schneider M, Minder C. Bias in metaanalysis detected by a simple, graphical test. BMJ 1997;315:629-34.

23 Kearney PM, Baigent C, Godwin J, Halls H, Emberson JR, Patrono C. Do selective cyclo-oxygenase- 2 inhibitors and traditional non-steroidal anti-inflammatory drugs increase the risk of atherothrombosis? Meta-analysis of randomised trials. BM/ 2006;332:1302-8.
24 Psaty BM, Kronmal RA. Reporting mortality findings in trials of rofecoxib for Alzheimer disease or cognitive impairment: a case study based on documents from rofecoxib litigation. JAMA 2008;299:1813-7.

25 Correction: report of specific cardiovascular outcomes of the ADVANTAGE trial. Ann Intern Med 2006:144:943.

26 Curfman GD, Morrissey S, Drazen JM. Expression of concern reaffirmed. N EnglJ Med 2006;354:1193.

27 Baron JA, Sandler RS, Bresalier RS, Lanas A, Morton DG, Riddell R, et al. Cardiovascular events associated with rofecoxib: final analysis of the APPROVe trial. Lancet 2008;372:1756-64.

28 Solomon SD, Wittes J, Finn PV, Fowler R, Viner J, Bertagnolli MM, et al. Cardiovascular risk of celecoxib in 6 randomized placebo-controlled trials: the cross trial safety analysis. Circulation 2008;117:2104-13.

29 Van Staa TP, Leufkens HG, Zhang B, Smeeth L. A comparison of cost effectiveness using data from randomized trials or actual clinical practice: selective cox-2 inhibitors as an example. PLoS Med 2009;6:e1000194.

30 Egger M, Juni P, Bartlett C, Holenstein F, Sterne J. How important are comprehensive literature searches and the assessment of trial quality in systematic reviews? Empirical study. Health Technol Assess 2003;7:1-76

31 Caldwell DM, Ades AE, Higgins JP. Simultaneous comparison of multiple treatments: combining direct and indirect evidence. $B M$ 2005;331:897-900

32 Hippisley-Cox J, Coupland C. Risk of myocardial infarction in patient taking cyclo-oxygenase-2 inhibitors or conventional non-steroidal anti-inflammatory drugs: population based nested case-control analysis. BMJ 2005;330:1366.

33 Cairns JA. The coxibs and traditional nonsteroidal anti-inflammator drugs: a current perspective on cardiovascular risks. Can J Cardiol 2007;23:125-31.

34 Wolfe F, Flowers N, Burke TA, Arguelles LM, Pettitt D. Increase in lifetime adverse drug reactions, service utilization, and disease severity among patients who will start COX-2 specific inhibitors: quantitative assessment of channeling bias and confounding by indication in 6689 patients with rheumatoid arthritis and osteoarthritis. J Rheumatol 2002;29:1015-22.

35 Chou R, Fu R, Carson S, Saha S, Helfand M. Methodological shortcomings predicted lower harm estimates in one of two sets of studies of clinical interventions. / Clin Epidemiol 2007;60:18-28.

36 Grosser T, Fries S, FitzGerald GA. Biological basis for the cardiovascular consequences of COX-2 inhibition: therapeutic challenges and opportunities. J Clin Invest 2006;116:4-15.

37 Fitzgerald GA. Coxibs and cardiovascular disease. $N$ Engl J Med 2004;351:1709-11.

38 Hermann M, Camici G, Fratton A, Hurlimann D, Tanner FC, Hellermann JP, et al. Differential effects of selective cyclooxygenase2 inhibitors on endothelial function in salt-induced hypertension. Circulation 2003;108:2308-11.

39 Sowers JR, White WB, Pitt B, Whelton A, Simon LS, Winer N, et al. The effects of cyclooxygenase- 2 inhibitors and nonsteroidal antiinflammatory therapy on 24-hour blood pressure in patients with hypertension, osteoarthritis, and type 2 diabetes mellitus. Arch Intern Med 2005;165:161-8.

40 Zhang J, Ding EL, Song Y. Adverse effects of cyclooxygenase 2 inhibitors on renal and arrhythmia events: meta-analysis of randomized trials. JAMA 2006;296:1619-32.

41 Harifforoosh S, Aghazadeh-Habashi A, Jamali F. Extent of renal effect of cyclo-oxygenase-2-selective inhibitors is pharmacokinetic dependent. Clin Exp Pharmacol Physiol 2006;33:917-24.

42 Zhang W, Jones A, Doherty M. Does paracetamol (acetaminophen) reduce the pain of osteoarthritis? A meta-analysis of randomised controlled trials. Ann Rheum Dis 2004;63:901-7.

43 Kuehn BM. FDA focuses on drugs and liver damage: labeling and other changes for acetaminophen. JAMA 2009;302:369-71.

44 Nuesch E, Rutjes AW, Husni E, Welch V, Juni P. Oral or transdermal opioids for osteoarthritis of the knee or hip. Cochrane Database Syst Rev 2009;4:CD003115.

Accepted: 08 October 2010 